POS PROCEEDINGS

Organization

of the XXII International Workshop

on Deep Inelastic Scattering and Related Subjects

(DIS2014)

XXII. International Workshop on Deep-Inelastic Scattering and Related Subjects,

28 April - 2 May 2014

Warsaw, Poland 


\section{Local Organizing Committee}

Barbara Badełek

Grzegorz Brona

Krzysztof Doroba

Jan Kalinowski

Małgorzata Kazana

Maria Krawczyk

Maciej Misiura

Andrzej Sandacz

Jakub Wagner

Aleksander Filip Żarnecki
University of Warsaw (co-chair)

University of Warsaw (co-chair)

University of Warsaw

University of Warsaw

National Centre for Nuclear Research

University of Warsaw

University of Warsaw

National Centre for Nuclear Research

National Centre for Nuclear Research

University of Warsaw

\section{Physics Programme Committee}

Barbara Badełek

Andrzej Bożek

Artur Kalinowski

Adam Kisiel

Krzysztof Kurek

Anna Stasto

Antoni Szczurek

Lech Szymanowski

Aleksander Filip Żarnecki
University of Warsaw (chair)

Institute of Nuclear Physics PAN

University of Warsaw

Faculty of Physics, Warsaw University of Technology

National Centre for Nuclear Research

Penn State and Institute of Nuclear Physics PAN

Institute of Nuclear Physics PAN

National Centre for Nuclear Research

University of Warsaw

\section{International Advisory Committee}

Halina Abramowicz

Sergio Bertolucci

Ian Brock

Allen Caldwell

Amanda Cooper-Sarkar

John Dainton

Cristinel Diaconu

Rolf Ent

Joel Feltesse

Stefano Forte

Elisabetta Gallo

Rolf-Dieter Heuer

Robert Klanner

Max Klein
Tel Aviv

CERN

Bonn

Munich

Oxford

Liverpool

Marseilles

Jefferson Lab

Saclay

Milano

Florence

CERN

Hamburg

Liverpool 


$\begin{array}{ll}\text { Aharon Levy } & \text { Tel Aviv (chair) } \\ \text { Lev Lipatov } & \text { St. Petersburg } \\ \text { Joachim Mnich } & \text { DESY } \\ \text { Hugh Montgomery } & \text { Jefferson Lab } \\ \text { Rosario Nania } & \text { Bologna } \\ \text { Fredrick Olness } & \text { Dallas } \\ \text { Juan Terron } & \text { Madrid } \\ \text { Robert Thorne } & \text { London } \\ \text { Katsuo Tokushuku } & \text { KEK } \\ \text { Steven Vigdor } & \text { BNL } \\ \text { Matthew Wing } & \text { London }\end{array}$

\section{Working groups and their conveners}

- WG1: Structure Functions and Parton Densities

Richard Ball Edinburgh

Vladimir Chekelian MPI Munich

Karol Kovarik Muenster

- WG2: Small- $x$, Diffraction and Vector Mesons

$\begin{array}{ll}\text { Mario Deile } & \text { CERN } \\ \text { Lidia Goerlich } & \text { Cracow } \\ \text { Stephane Munier } & \text { Palaiseau }\end{array}$

- WG3: Electroweak Physics and Beyond the Standard Model

$\begin{array}{ll}\text { Maxime Gouzevitch } & \text { Lyon } \\ \text { Anna Kaczmarska } & \text { Cracow } \\ \text { Milada Margarete Muehlleitner } & \text { Karlsruhe } \\ \text { Krzysztof Turzynski } & \text { Warsaw }\end{array}$

- WG4: QCD and Hadronic Final States
Vladimir Braun
Regensburg
Daniel Britzger
DESY
Konstantinos Kousouris CERN 
- WG5: Heavy Flavours

$\begin{array}{ll}\text { Ivan Belyaev } & \text { Moscow } \\ \text { Martin Gorbahn } & \text { Liverpool } \\ \text { Anze Zupanc } & \text { Ljubliana }\end{array}$

- WG6: Spin Physics

$\begin{array}{ll}\text { Yann Bedfer } & \text { Saclay } \\ \text { Barbara Pasquini } & \text { Pavia } \\ \text { Ernst Sichtermann } & \text { LBNL }\end{array}$

- WG7: Future experiments

Abhay Desphande

Alexei Prokudin

Alice Valkarova
Stony Brook

JLab

Prague 